University of New Mexico

UNM Digital Repository

Biology Faculty \& Staff Publications

Academic Department Resources

$1-1-1981$

\title{
Sex ratio evolution in a variable environment
}

Eric Charnov

Hartogh R.L. Los-den

W.T. Jones

J. van den Assem

Follow this and additional works at: https://digitalrepository.unm.edu/biol_fsp

Part of the Biology Commons

\section{Recommended Citation}

Charnov, E.L., R.L. Los-den Hartogh, W.T. Jones and J. van den Assem. 1981. Sex ratio evolution in a variable environment. Nature 289:27-33

This Article is brought to you for free and open access by the Academic Department Resources at UNM Digital Repository. It has been accepted for inclusion in Biology Faculty \& Staff Publications by an authorized administrator of UNM Digital Repository. For more information, please contact disc@unm.edu. 
Received 4 August; accepted 22 September 1980.

1. Charnov, E. L. Am. Nat. 113, 465-480 (1979).

2. Maynard Smith, J. The Evolution of Sex (Cambridge University Press, 1978).

3. Williams, G. C. Sex and Evolution (Princeton University Press, 1975)

4. Charnov, E. L., Maynard Smith, J. \& Bull, J. J. Nature 263, 125-126 (1976).

5. Charnov, E. L. Proc natn. Acad. Sci. U.S.A. 76, 2480-2484 (1979).

6. Warner, R. R. Am. Nat. 109, 61-82 (1975).

7. Warner, R. R., Robertson, D. R. \& Leigh, E. G. Science 190, 633-638 (1975).

8. Leigh, E. G. Jr, Charnov, E. L. \& Warner, R. R. Proc. natn. Acad. Sci. U.S.A. 73, 3656-3660 (1976).

9. Ghiselin, M. T. Q. Rev. Biol. 44, 189-208 (1969).

10. Ghiselin, M. T. The Economy of Nature and the Evolution of Sex (University of California Press, 1974).

11. Hoagland, K. E. Malacologia 17, 365-391 (1978)

12. Charnov, E. L. Am. Nat. 113, $715-734$ (1979).

13. Lloyd, D. G. N. Z. J. Bot. 17, 595-606 (1979).

14. Charlesworth, B. \& Charlesworth, D. Am. Nat. 112, 975-997 (1978)

15. Willson, M. F. Am. Nat. 113, 777-790 (1979).

16. Bawa, K. S. Ann. Rev. Ecol. Syst. 11 (in the press)

17. Maynard Smith, J. Am. Scient. 64, 41-45 (1976).

18. Fisher, R. A. The Genetical Theory of Natural Selection (Oxford University Press, 1930)

19. Hamilton, W. D. Science 156, $477-488$ (1967).

19. Hamilton, W. D. Science 156, $477-488$ (1967).
20. Trivers, R. L. \& Hare, H. Science 191, 249-263 (1976).

21. Charnov, E. L. Am. Nat. 112, 317-326 (1978).

22. Assem, J. van den Neth. J. Zool. 21, 373-402 (1971)

23. Chweyreuv, I. C. r. Séanc. Soc. Biol. 74, 695-699 (1913).

24. Sandlan, K. Ecol. Ent. 4, 365-378 (1979).
25. Clausen, C. D. J. N. Y. ent. Soc. 47, 1-9 (1939).

26. Holdaway, F. T. \& Smith, H. F. Aust. J. exp. Biol. med. Sci. 10, 247-159 (1933).

27. Brunson, M. H. Science 86, 197 (1937).

28. Nozato, K. Kontyu 37, 134-146 (1969).

29. Assem, J. van den Neth. J. Zool. 20, 329-352 (1970).

30. Assem, J. van den in Sluipwespen in relatie tot hun gastheren (eds Klomp, H. \& Wiebes, J. T.) 64-96 (Centrum voor Landbouwpublicaties en documentatie, Wageningen, 1979).

31. Arthur, A. P. \& Wylie, H. G. Entomophaga 4, 297-309 (1959).

32. Ryan, R. B. \& Rudinsky, J. A. Can. Ent. 94, 748-763 (1962).

33. Berry, P. A. J. Econ. Ent. 32, 717-721 (1939).

34. Kishi, Y. Appl. ent. Zool. 5, 126-132 (1970).

35. Trivers, R. L. \& Willard, D. E. Science 179,90-92 (1973)

36. Hamilton, W. D. in Sexual Selection and Reproductive Competition in Insects (eds Blum, $\mathbf{M}$ S. \& Blum, N. A.), (Academic, New York, 1979).

37. Kirkpatrick, R. L. \& Wilbur, D. A. J. econ. Ent. 58, 979-985 (1965).

38. Krombein, K. V. Trap Nesting Wasps and Bees (Smithsonian, Washington, DC, 1968).

39. Stephen, W. P. \& Osgood, C. E. J. econ. Ent. 58, 965-968 (1965).

40. Baroni Urbani, C. in Social Insects Vol. 1 (ed. Hermann, H. R.) 19-121 (Academic, New York, 1979).

41. Hamilton, W. D. J. theor. Biol. 7, 1-52 (1964)

42. Charnov, E. L., Gotshall, D. \& Robinson, J. Science 200, 204-206 (1978).

43. Gregg, K. Selbyana 1, 101-113 (1975).

44. Peterson, J. J. J. Nematol. 4, 83-87 (1972).

45. Poinar, G. O. Jr Nematodes for Biological Control of Insects (CRC Press, Boca Raton, Florida, 1979)

46. Shapiro, D. Y. Adv. Study Behav. 10, 43-102 (1979).

47. Charnov, E. L. \& Bull, J. J. Nature 266, 828-830 (1977)

48. Freeman, D. C., Harper, K. T. \& Charnov, E. L. Oecologia (in the press).

\title{
Sex ratio evolution in a variable environment
}

\author{
E. L. Charnov", R. L. Los-den Hartogh ${ }^{\dagger}$, W. T. Jones ${ }^{\ddagger}$ \& J. van den Assem ${ }^{\dagger}$ \\ * Department of Biology, University of Utah, Salt Lake City, Utah 84112 \\ † Zoölogisch Laboratorium, Rijks Universiteit Leiden, Leiden, The Netherlands \\ ‡ Department of Biological Sciences, Purdue University, West Lafayette, Indiana 47907
}

We develop a natural selection model for sex ratio control in a spatially variable environment. Predictions of sex ratio alteration as a function of environmental change are tested in laboratory experiments with two parasitic wasps. Field data from a variety of other organisms also support the model. Finally, we discuss possibilities and difficulties for testing this type of evolutionary model.

SEX allocation theory deals with the impact of natural selection on the allocation of resources to male compared with female reproductive function ${ }^{1}$. For dioecious species the problem of interest is the evolution of the sex ratio; for a simultaneously hermaphroditic species, it is the allocation to sperm versus eggs in each breeding season ${ }^{2-5}$; for a sequential or sex-reversing hermaphrodite, the questions are the sex order (male or female first?) and the proportion of an individual's lifetime devoted to each $\operatorname{sex}^{6-12}$. The theory also gives the conditions for a sexual state to be evolutionarily stable. For example, it specifies when some form of hermaphroditism is favoured over dioecy ${ }^{1-16}$.

It was previously ${ }^{1}$ shown that many sex allocation problems have the property that the equilibrium or $\mathrm{ESS}^{17}$ condition satisfies a particular optimality principle. The allocation to male versus female function, which is favoured by natural selection, can often be shown to maximize the fitness gains through male function multiplied by the gains through female function ${ }^{1}$. From this product formalism, one can derive the classic results of sex ratio theory. Examples are the 'equal resource into each sex' theorem of Fisher ${ }^{18}$, the 'local mate competition' results of Hamilton ${ }^{19}$ and several results from the Trivers-Hare theory for eusocial Hymenoptera ${ }^{20,21}$. Here, we shall apply the formalism to sex ratio evolution in a variable environment and will provide a set of laboratory tests for the resulting predictions. As the organisms which stimulated our modelling efforts were parasitic wasps, we shall develop the model with reference to their life histories.

\section{Parasitic wasps in a variable environment}

Imagine an outcrossing parasitic wasp which has discrete generations and where the females attack hosts over a wide range of sizes ${ }^{22-34}$. A single egg is laid on each host. The host is paralysed or killed by a sting from the female wasp, so that the total food for her offspring's development is contained in the host at the moment of attack. If the host is small, the wasp larva will have relatively little food and will emerge as a small adult. If 
the host is large, the resulting wasp will be large. Suppose further that the reproductive consequences of being a large compared with a small adult wasp differ, depending on whether the individual is a male or a female. For example, over her lifetime, a large female may lay 10 times as many eggs as a small female; however, a large male may be only three times as effective as a small male at inseminating females. As the female wasps lay eggs in a variety of host sizes, the environment is thus variable with respect to opportunities for production of sons versus daughters. The mother is assumed to control her sex ratio (proportion male eggs) as a function of host size. Haplodiploid sex determination provides her with a physiological mechanism for this control. Our interest is in predicting what sex ratio she will produce in a given host size. To do this, we first specify the host size distribution and the expected reproductive success for an adult wasp of a given sex and size.

Let $f(x)$ be the probability density function for hosts of size $x$ which are attacked by the female. For simplicity, assume $x$ to be continuous. Let $W_{1}(x)$ be the relative fitness of a son derived from a host of size $x$. A male's fitness is measured relative to other males ${ }^{1}$, so that $W_{1}(x)$ scales the ability of a male of size $x$ to inseminate females over his lifetime. Let $W_{2}(x)$ be the lifetime egg production of a female derived from a host of size $x$. Both these fitness measures include survivorship to adulthood, which may vary with host size ${ }^{22}$. Finally, let $r(x)$ be the proportion of unfertilized or male eggs laid in host size $x, r(x)$ is controlled by the mother wasp. Using techniques from population genetics, one can show that the equilibrium or $\operatorname{ESS}^{17} r(x)$ satisfies the following product relationship ${ }^{1}$. It is the function, $r(x)$, which maximizes the product of the fitness gained through the decisions to produce sons and that gained through the decisions to produce daughters, or:

Maximize

$\left[\int_{x} f(x) W_{1}(x) r(x) \mathrm{d} x\right] \times\left[\int_{x} f(x) W_{2}(x)(1-r(x)) \mathrm{d} x\right] 0 \leqslant r(x) \leqslant 1$

If we impose the condition that a large daughter gains more in relative fitness than a large son $\left[W_{2}(x) / W_{1}(x)\right.$ increases with $\left.x\right]$, the solution to equation (1) reduces to a very simple form ${ }^{1,35}$. Only sons should be produced in small hosts and only daughters in large ones. There is a certain host size $(\tau)$ where the switch from sons to daughters occurs (Fig. 1a). Equation (1) now takes the form:

Maximize

$$
\left[\int_{0}^{\tau} f(x) W_{1}(x) \mathrm{d} x\right] \times\left[\int_{\tau}^{\infty} f(x) W_{2}(x) \mathrm{d} x\right]
$$

Although the model predicts a threshold $(\tau)$ for the sex ratio change, it is unlikely that biological data would show such an abrupt transition. A more likely pattern would be that of all sons in small hosts and daughters in large, with a gradual sex ratio transition in between (illustrated in Fig. $1 a)^{1,35}$. We expect a gradual transition for two reasons. First, the model assumes time constant fitness and host distribution values. If these vary through time, then so does $\tau$. The quality of a host may also depend somewhat on factors which cannot be known to the mother when she deposits an egg. Selection would then favour a more gradual shift in sex ratio. However, a second reason is that even if the mother wasp made perfect decisions, the experimenter may not be able to classify the hosts perfectly with respect to criteria used by the wasps. For example, differentiation of equation (2) with respect to $\tau$ shows that the hosts are theoretically to be classified by the ratio $W_{2}(x) / W_{1}(x)$. Those with low ratios are son hosts, those with large are daughter hosts. Let $k$ be the threshold ratio. Now, suppose that the wasp makes perfect decisions, with all hosts above $k$ being given to daughters. If the experimenter, however, uses a measure of host size $(\hat{x})$ which is imperfectly correlated with the $W_{2}(x) / W_{1}(x)$ ratio, we will not see the threshold. The experiment would show a gradual shift in sex ratio as a function of $\hat{x}$, our measure of host quality.
In addition to the sex ratio shift with host size, the model makes one other general type of prediction. The concepts of large and small host as discussed above have no absolute meaning. That is, a host is only large or small relative to the other hosts being attacked. To calculate theoretically whether a host is to be a son or daughter host, we must first know the host size distribution which is being attacked. We expect to find an increasing fraction of daughters in larger hosts; however, what constitutes larger may vary from place to place, or time to time. Consider the host marked $z$ on Fig. $1 b$. If the distribution of host sizes is $\mathrm{i}, z$ is a large host. If the distribution is $\mathrm{ii}, z$ is a relatively small host. Host $z$ should contain a greater proportion of daughters when large (case i) than when small (case ii).

Two other factors implicit in the model deserve further comment. First, the sex ratio under discussion is the ratio at the egg stage. Data often show that survivorship declines in small hosts ${ }^{22}$. If this decline is different for the two sexes, the differential mortality as a function of host size would itself generate a sex ratio shift among emerging wasps, independently of a true sex ratio shift at the egg stage. However, such differential mortality would also contribute to natural selection favouring a shift by the mother wasp. Some evidence indicates that daughters may sometimes be unable to reach pupation size in very small hosts (a pupal parasite of Lepidoptera ${ }^{34}$ ), but even here a strong sex ratio shift at the egg stage with host size could be demonstrated. The relativity prediction discussed previously is a good control for

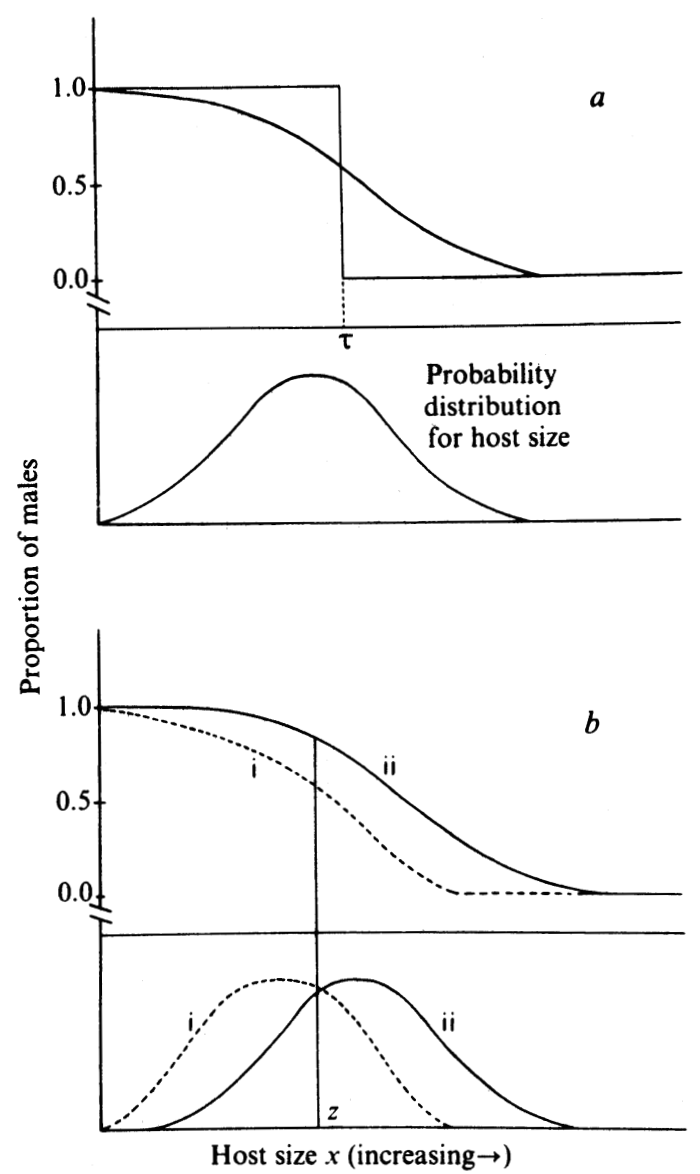

Fig. 1 Sex ratio as a function of host size, $a$, Provided females gain more fitness by being larger than do males (that is, $W_{2}(x) / W_{1}(x)$ increases with $x$ ), the sex ratio is predicted (equation (2)) to be female biased in large hosts, male biased in small. There is a threshold host size $(\tau)$ where a change-over occurs. For reasons discussed in the text, the expected pattern is not a threshold, but more gradual sex ratio shift. $b$, Whether a given host is to be a son host or a daughter host depends on the entire host size distribution. Host $z$ is a relatively large host if the host distribution is $i$; it is a small host if the distribution is ii. It should contain more males when it is small. Host size distributions are simply illustrative. 
Fig. 2 Sex ratio in Lariophagus. The proportion of males emerging is plotted against the size of the hosts (weevil larvae) (tunnel width) which were offered in a 'carousel experiment'. Curve $b$ results when female wasps are presented sequentially with 20 hosts of a single size. Curves $a$ and $c$ result when wasps are presented with an alternating sequence of two host sizes, where the hosts are $0.4 \mathrm{~mm}$ different is size. Curve $c$ is where the host of interest was the smaller of the two. For example, hosts of $1.4 \mathrm{~mm}$ were offered alone and gave a sex ratio of $15 \%$ males (curve $b$ ). When they were offered alternately with $1.8-\mathrm{mm}$ hosts, they gave a sex ratio of $30 \%$ males (curve $c$ ). When offered with $1.0-\mathrm{mm}$ hosts, their sex ratio dropped to $2 \%$ males (curve a). The number of emerging wasps is given for each data point. The experimental technique is as follows: the weevil Sitophilus is cultured on wheat grains at $25^{\circ} \mathrm{C}$. Twice a week freshly emerged weevils are placed on fresh (unfrozen) grain for oviposition and removed again 7 days later. Grains with the necessary age range of larvae can then be irradiated with $X$ rays to measure the exact size of the larvae by measuring tunnel diameter ${ }^{37}$. The grains are then stored at $10^{\circ} \mathrm{C}$ for up to 14 days. At $10^{\circ} \mathrm{C}$ the larvae do not grow and only a small percentage die. Grains with larvae of a desired size are picked out and used in

carousel experiments. The oviposition carousel (described in ref. 30) makes it possible to offer individual female wasps grains with known contents for a specified period of time in a specified order. Inseminated females are isolated from cultures on the day of their emergence and stored for $24 \mathrm{~h}$ at $18-20^{\circ} \mathrm{C}$. They are used in a carousel experiment, with a series of 20 grains. Each grain is offered to a female for $2.5 \mathrm{~h}$, then labelled and stored at $25^{\circ} \mathrm{C}$ until a weevil or a wasp emerges. If there is no emergence, the grain is opened to see what happened to the weevil larvae. The head width was measured for all wasps.

this effect, as the shift is predicted within a single host size. The model also predicts that the overall sex ratio should be biased towards males. However, this is again sex ratio at the egg stage. If survivorship declines with host size, then at emergence the overall sex ratio may be female biased (because males are put in small hosts).

The second factor is the assumption that the species is outbred. Hamilton ${ }^{19.36}$ has developed a theory for certain forms of inbreeding, or nonrandom competition among males for mates, which predicts a generally female-biased sex ratio. In the most extreme cases, all females in a brood are inseminated by their brothers. Here, theory predicts (and data strongly support) that a mother wasp should produce mostly daughters. This 'local mate competition' (LMC) is thus a factor which may alter the overall sex ratio in the direction of females. Although we cannot eliminate it as a factor in our wasp systems, it seems unlikely as a general explanation for a host shift in sex ratio. Long-term ( $\sim 20$ yr or 200 generations) laboratory cultures of one species studied here 22 consist of hundreds of wasps mixed together. There is much mixing of the offspring of individual females. Such conditions are quite the opposite of those postulated by Hamilton and would select against LMC effects. In this same species (Lariophagus), other experiments presented the wasps with groups of hosts and sometimes put two or more female wasps together with a batch of hosts. The present experiments presented a single female with one host at a time and they gave essentially the same results as the former. With a strong LMC model, the wasps would be predicted to behave differently in the two conditions. For these reasons, we have derived the predictions from a model with outbreeding (Fig. 1).

\section{On testing the model}

A general test of the model would consist of showing that sex ratio varied as a function of host size, and that the daughter/son fitness comparison also changed with host size. Both would have to change in the predicted directions. We would also have to show that differential mortality or LMC could not account for the results. The predicted relative nature of the sex ratio shift itself provides a powerful, qualitative test of the hypothesis. There are at least three ways in which we might test this prediction.

(1) Geographical variation. If in different places the wasp species is associated with different host size distributions, this natural variation can be turned into a test. For instance, if the same host is present in both locations, but is a small host in one place and is large in the other, a sex ratio shift is expected in that host.

(2) Laboratory selection experiments. It should be possible to mimic the effects of geographical variation in the laboratory. If the parasitoids can be reared on only small hosts, or only large hosts, sex ratio shifts through time (over several generations) are expected, if the sex ratio is a heritable character. The small hosts should shift from producing mostly males towards a more equal sex ratio. The large hosts should shift from mostly females towards a more equal ratio.

(3) Temporal variation. If the wasps naturally confront host size distributions which change from generation to generation, they might have the ability to alter their sex ratio on a short time scale, in response to the prevailing distribution. The most reasonable source for a shift in host size utilization would be a shift in host abundance. Because small hosts are poorer for offspring, a mother might be expected to ignore them when confronted with an abundance of larger hosts. This behavioural shift would require the wasps to pick up, store and use information about the distribution. However, if the natural host size distribution was either constant through time, or continued to change within a wasp generation, we might expect the wasps not to respond to short-term shifts in the distribution. We would then require geographical variation or a laboratory selection experiment to test the relativity idea.

We report here the results of a series of experiments on this host size model. Our experiments relate to the abilities of two parasitoid species to alter their sex ratio decisions on a short time scale. We tested the basic model by looking at sex ratio as a function of host size. We then confronted wasps with altered 


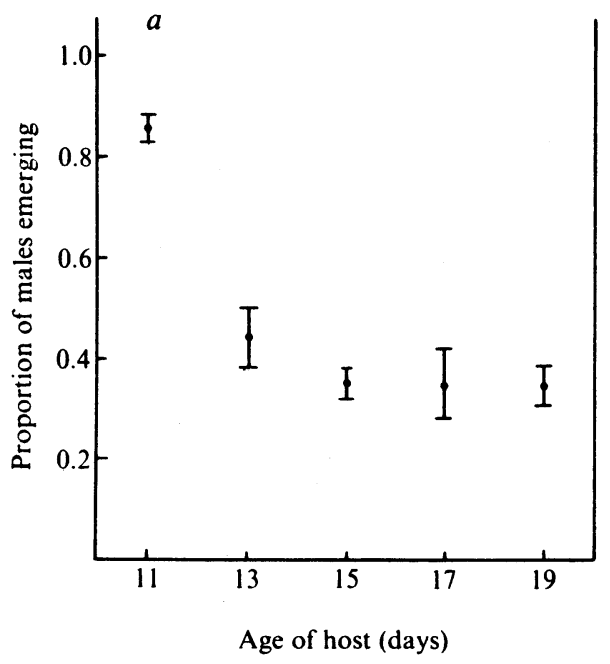

Age of host (days)

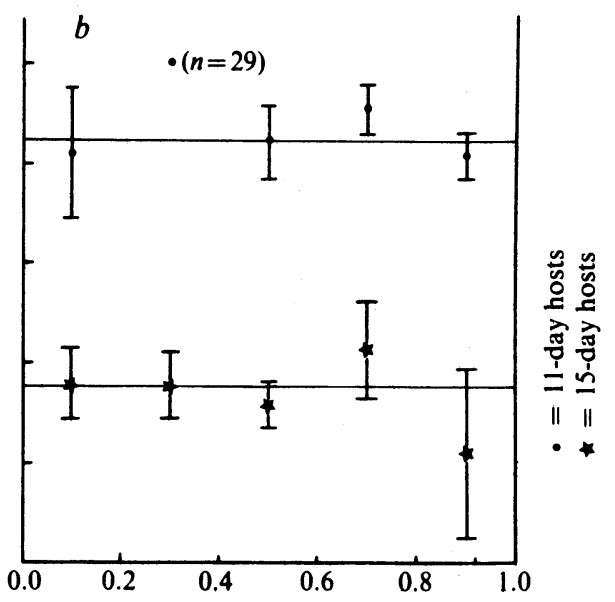

Fig. 3 Sex ratio as a function of host age for Heterospilis attacking Callosobruchus. $a$, Wasps presented with a single-aged host for a period of $24 \mathrm{~h}\left(30^{\circ} \mathrm{C}\right.$ and $70 \%$ relative humidity, host density at $\sim 20$ hosts per female wasp). Data given are means \pm 2 s.e. $b$, Wasps were in an environment consisting of 11 - and 15-day hosts presented simultaneously. In the five experiments, the proportion of 11-day hosts varied from 0.1 to 0.9 . Data are the mean sex ratios within each host age $( \pm 2$ s.e.). The lines in $b$ refer to the proportion of males found in $a$. host size distributions and measured the sex ratio shift. Our data indicate that large wasps come from large hosts and that both males and females gain increased reproductive success by being large.

\section{Sex ratio in Lariophagus and Heterospilis}

Lariophagus distinguendus is a small $(1-3 \mathrm{~mm})$ parasitic wasp (family Pteromalidae) which attacks larvae of the common granary weevil, Sitophilus granarius ${ }^{22,29,30}$. The weevils oviposit in grains of wheat, and a single larva develops inside the grain. The parasite stings the larva and deposits a single egg. An adult wasp emerges about 18 days later (at $25^{\circ} \mathrm{C}$ ). The wasp will parasitize a variety of host sizes ${ }^{22}$. The larva hollows out the grain as it develops. Size of weevil larvae is correlated with age, but the relationship is imprecise. For the present experiments we measured host size directly. The size of the weevil larva is strongly correlated with the diameter of its tunnel ${ }^{37}$. Tunnel diameter was measured on $\mathrm{X}$-ray photographs ${ }^{22,37}$.

Heterospilis prosopoidis is a small $(1-3 \mathrm{~mm})$ parasitic wasp in the family Braconidae. It has a similar life history to that of Lariophagus in that it lays a single egg on the larvae of weevils, attacking a variety of host sizes. It was studied here attacking the azuki bean weevil, Callosobruchus chinensis. At $30^{\circ} \mathrm{C}$, weevil development (egg to adult) takes about 21 days. Weevil larvae 11-19 days old are accepted by the wasp. Experiments with this system used weevil age as a measure of host size.

Van den Assem ${ }^{22}$ had previously shown, using weevil age as a measure of size, that Lariophagus adjusts its sex ratio as a function of weevil size. Mostly males were produced in young weevils, mostly females in older weevils. His experiments were carried out by presenting wasps with weevils of only one age class. The experiments were also controlled to show that the observed sex ratio shift could not be due to differential, sexspecific mortality (even though young weevils produced a much higher death rate). The data also suggested that the wasp used a relative measure of host size.

We have repeated these earlier ${ }^{22}$ experiments, using the more exact measure for host size. Figure $2 b$ shows the results. As expected, sons are produced in small hosts, daughters in large. A second experiment consisted of presenting wasps with two host sizes, offered in equal abundance. The hosts were always $0.4 \mathrm{~mm}$ different in size and the wasp was presented with an alternating sequence (large, small,...). The data from these two experiments thus consist of a given host size in three situations: alone, smaller of two sizes and larger of two sizes. For example, we have $1.4-\mathrm{mm}$ hosts alone, and paired with $1.0-$ or with $1.8-\mathrm{mm}$ hosts. If we consider some host size $z$, it is clear that the three experiments ( $z$ small, $z$ alone, $z$ large) represent successively more left-shifted host size distribution curves. This corresponds to a shift from distribution ii to distribution $\mathrm{i}$ in Fig. $1 b$. If the wasp is adapted to alter its sex ratio on a short time scale, it should put relatively more daughters into host $z$ as we move across the three treatments.

Curve $a$ in Fig. 2 represents the sex ratio as a function of host size, when the host represented was the larger of the two hosts (for example, 1.0 was paired with 0.6 ). Note that every point lies below (is more female biased) the curve for the hosts when they were by themselves. Curve $c$ (Fig. 2) shows the corresponding relationship for hosts where they were the smaller of the two hosts (for example, 1.0 was paired with 1.4). With a single exception, $0.7 \mathrm{~mm}$, this curve lies above (more male biased) the other two curves. We have not provided statistical tests for the individual host sizes because the hypothesis relates to the general order of the curves. It is indeed remarkable that, even given small (10-30) to moderate (50-100) sample sizes, the curves preserve their theoretically predicted order, over the entire range of host sizes. This is the strongest evidence for the shift predicted by sex ratio theory.

Using host age as a measure of size, one of us (W.J.T.) carried out a similar set of experiments with Heterospilis. Presenting wasps with a single host size produced the relationship in Fig. $3 a$. Small hosts (11 days) were very male biased, whereas larger hosts were more female biased. A series of control experiments showed that this result could not be due to differential, sexspecific mortality. To see if these wasps could also adjust the sex ratio to a shifting host environment, we carried out a second set of experiments with just two host ages (11 and 15 days, or 15 and 19 days), presented in equal numbers. In comparison with the experiments in which the wasp received only one host size, the 11- and 15-day hosts now showed no altered sex ratio. Indeed, the 15-day hosts showed the same sex ratio when alone, or when combined with either 11- or 19-day hosts. Moderate to large sample size (88-1,024 emerging wasps per host size) makes these results quite reliable. Only one predicted sex ratio shift was seen. When 19-day hosts were presented alone, they gave a proportion of males of $0.36(n=786)$. However, when they were presented with equal numbers of 15-day hosts, the sex ratio dropped to $0.25(n=88)$. Such a shift is statistically significant (one-sided, Fisher exact test; $P=0.03$ ) in the predicted direction.

We carried out a further set of experiments with Heterospilis. These involved 11- and 15-day hosts, presented to the wasp in various proportions (proportions of 11-day hosts $0.1,0.3,0.5$, $0.7,0.9)$. As can be seen in Fig. $3 b$, the wasp failed in 8 out of 10 cases to show any deviation (significant at the 0.05 level) from the sex ratio predicted from the experiments with only one host size. Clearly, there is no pattern of sex ratio shift with changing host proportions (at least for 11- and 15-day hosts). Although 
Heterospilis does not immediately adjust its sex ratio to host size distributions, it is still possible that its offspring sex ratios are attuned to host distributions in a way predicted by the model. It may be that the host size distributions naturally encountered by Heterospilis do not vary greatly between wasp generations or that they vary within generations, and thus these wasps would not be expected to respond to short-term shifts in the distribution. At least for this species, a selection experiment or geographic comparison seems to be necessary to test the model further.

At least two other parasitic wasps which show a sex ratio shift with host size also show short-term adjustment of the sex ratio $^{23,24}$. Both species overproduce daughters in large hosts, sons in small. Chewyreuv ${ }^{23}$ studied parasitoid wasps of the genus Pimpla (family Ichneumonidae) which attack lepidopteran pupae. When he offered a female wasp a mixture of host pupae consisting of Sphinx (large) and Piéris (small), the wasp produced mostly females in the large, males in the small. However, when the Piéris pupae were alternated with those of Vanessa (still smaller), the same wasp produced female offspring in the former and males in the latter. Sandlan ${ }^{24}$ also studied a pupal parasite of Lepidoptera, the wasp Coccygomimus turionella. Here, individual wasps confronted with a single host size gradually altered the sex ratio through time. If the single size was large, the sex ratio declined from being female biased to being more male biased. A small size showed the opposite change-male biased towards more female biased. All these sex ratio shifts are in the theoretical direction.

\section{Fitness as a function of host size}

As the sex ratio shift is towards daughters in larger hosts, a further test of the theory consists of determining whether a female gains more fitness by being large than does a male. Measuring individual fitness is very difficult, especially for males, where the value must reflect the relative ability to gain access to females. Using Lariophagus, a set of experiments was designed to investigate whether wasp size was correlated with the size of its host, and also whether increased wasp size became translated into increased individual fitness. Figure 4 shows the result of rearing almost 2,000 individuals. Clearly, larger weevils give rise to large wasps. Two facts about Fig. 4 are of particular interest. First, the relationship is different for males and females. From the same-sized host, males are on average smaller than the corresponding female. This presumably reflects the fact that males show a shorter developmental time to adulthood on any given host size ${ }^{22}$. Second, both curves show a steep linear increase over the weevil size range $0.7-1.3 \mathrm{~mm}$. At $1.3 \mathrm{~mm}$ both show an abrupt transition. Average male size no longer increases with weevil size, whereas average female size goes up at a much reduced rate (slope of curve here is about $12 \%$ the previous slope).

Figure 5 shows that increased size translates into both increased male and increased female fitness. For females the measure is the lifetime production of eggs, for males it is length of life. This is not a complete measure of male fitness, because it gives no indication of male mating ability. Small males have no difficulty in getting females to accept them as mates ${ }^{22}$. Because females typically mate once for life, small males would seem to give adequate numbers of sperm, but we do not know how such small males fare in mate competition with larger males.

A similar set of experiments with female Heterospilis showed that lifetime fecundity increased about 20 times, comparing 11-day with 15-day hosts [1.3 offspring $($ s.d. $=2.87, n=61)$ compared with 27.9 (s.d. $=8.38, n=46)]$. Interestingly, males from the same-aged hosts showed a much smaller fitness size effect, the measure here being females inseminated over a 3-h period (with 20 females available). Eleven-day males inseminated an average of 2.8 females (s.d. $=2.91, n=38$ ), compared with 8.3 (s.d. $=2.36, n=31$ ) for 15-day males. Although these data suggest that daughters gain more by being large than do sons, they are not conclusive. It would be very useful to know how individual size affects other fitness components such as female search ability or female ability to attack large hosts.

There is another type of data which bear on this fitness gain issue, at least for Lariophagus. If fitness within each sex is related to the size of the wasp, host sizes over the range $1.3-2.0 \mathrm{~mm}$ show very little change in relative female/male size (Fig. 4), whereas relative female/male size is increasing over the host size range $0.6-1.3 \mathrm{~mm}$. We might then expect that host sizes above 1.3 would be treated quite similarly with respect to sex ratio. As shown in Fig. $2 b$ (host size alone experiments), the proportion of sons drops rapidly with increasing host size until a host size of $1.3 \mathrm{~mm}$. The sex ratio is about 0.17 males at this host size, but it does not become more female biased as we move to even larger hosts. The experiments presented in Fig. 2 show that the wasp can alter its sex ratio when a two-host environment consists of both hosts above $1.3 \mathrm{~mm}$. However, these shifts are small relative to the alteration in sex ratio observed over a host size range of $0.6-1.3 \mathrm{~mm}$. The sex ratio versus host age relationship measured earlier by van den Assem ${ }^{22}$ shows a similar abrupt transition in sex ratio at the host age of 23-24 days. X-ray calibration shows that this age corresponds to a mean host size close to $1.3 \mathrm{~mm}$.

\section{Other hymenopteran systems}

There are other parasitoid systems where a range of host sizes is attacked, and where sex ratio alters with host size 22-34 $^{\text {The shift }}$ is always in the direction of more females from larger hosts. There are other hymenopteran systems which show similar trends. 'Trap nesting' (ref. 38) refers to free-living bees and wasps where the mother places food plus an egg in a cell constructed inside a crevice (for example, a hollowed-out twig). The size of the crevice places constraints on the cell size, the amount of food packed in and consequently the size of the resulting adult wasp ${ }^{38,39}$. Trap nesters have been much studied because they will commonly nest in soda-straws, holes drilled in wood blocks, or other man-made crevices ${ }^{38,39}$. Because of the importance of some trap-nesting bee species for agricultural pollination ${ }^{39}$, many data now exist. Figure 6 shows the sex ratio (proportion of emerging males) as a function of hole diameter in artificial nesting material for 11 species of bees and wasps. With one exception, these data are from the book by Karl Krombein ${ }^{38}$. This documents no trap nester with a reverse sex ratio shift, although several species show evidence of no sex ratio shift for the hole sizes presented (several wasps of the genera Stenodynerus and Ancistrocerus). A reverse shift might be expected in species where the males show aggression and territoriality with respect to mating ${ }^{35,40}$. Here, increased size could

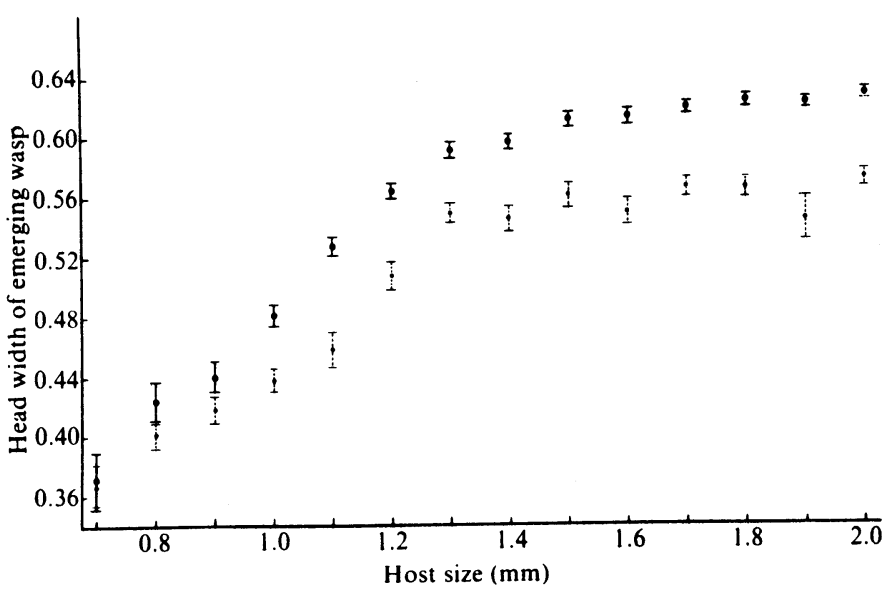

Fig. 4 Size of Lariophagus related to its host size (tunnel width). Means $( \pm 1$ s.e.) of head width of males (lower curve) and females (upper curve) are plotted against host weevil size. Experimental details are given in Fig. 2 legend. Least squares regressions calculated on the means: (1) $\delta$, host sizes $0.7-1.3 \mathrm{~mm}, y=0.16+0.28 x, r=0.98, P<0.001$. (2) $\delta$, host sizes 1.4$2.0 \mathrm{~mm}$, correlation not significant at 0.05 level. (3) $q$, host sizes $0.7-1.3 \mathrm{~mm}$, $y=0.11+0.37 x, r=0.99, P<0.001$. (4) $\&$, host sizes $1.4-2.0 \mathrm{~mm}, y=$ $0.54+0.044 x, r=0.92, P<0.01$. 

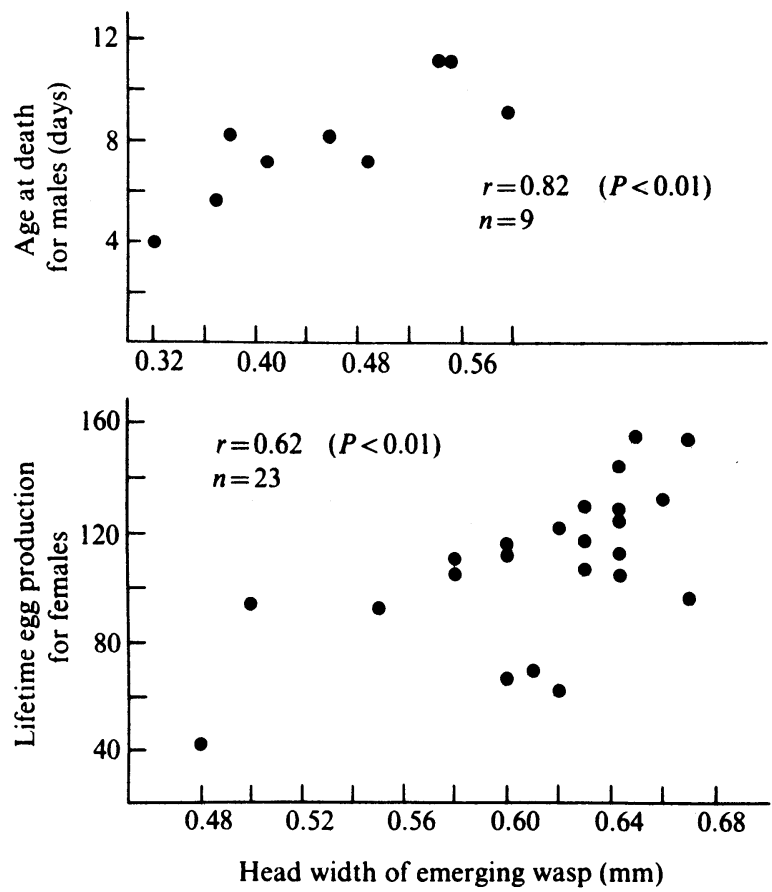

Fig. 5 Fitness as a function of individual size in Lariophagus. Both males and females gain by being large. Females were presented with an abundance of hosts. Experiments were run at $18^{\circ} \mathrm{C}$.

be translated into relatively greater male reproductive success ${ }^{35}$. It would be very interesting to know if any trap-nesting species shows geographical variation in the hole-size distribution. Such variaton should correlate with sex ratio shifts within a specified hole size, similar to those predicted (Fig. $1 b$, host size $z$ ) for parasitoids. However, the situation for trap nesters is somewhat more complicated than the simple model developed here. At least two other sex ratio effects occur with the hole-size shifts. There are often several cells placed in the same crevice-that is, a straw will contain a sequence of offspring. Because males typically emerge before females, it is common for the mother to put more daughters in the deep cells, more sons near the opening $^{38}$. In this way, the sons can get out first without having to destroy the wasp in front. Except where different mothers may supersede each other at a particular hole, the offspring in a given hole will be siblings. Some degree of cooperation may be expected ${ }^{41}$, but the parents should benefit by using deep crevices for a mixture of sons and daughters. The other factor affecting sex ratio is that a son costs (in the sense of Fisher ${ }^{18.21}$ ) less than a daughter to produce. They require smaller cells (regardless of the diameter of the hole) and less food ${ }^{38}$. The equilibrium sex ratio should reflect this lowered cost through production of more males ${ }^{20,21}$. Even though these two factors complicate the situation, the hole-size effect, as illustrated in Fig. 6, still seems to be a dominating factor.

\section{Discussion}

Various biological systems in addition to Hymenoptera show sex ratio shifts of the type discussed here. These include pandalid shrimp ${ }^{12,42}$, various orchids ${ }^{43}$, entomogenous nematodes ${ }^{44,45}$ and molluscs of the genus Crepidula ${ }^{11}$. In these cases, larger individuals typically become females, smaller ones male. Some of these species are sex reversers (sequential hermaphrodites), beginning life as a male and later changing into a female. Of course, many sex reversers go in the other direction; particularly among coral reef fish, species are known where individuals begin life as females, and become males when they are large enough to compete for a breeding territory ${ }^{6-10,46}$.
As developed here and elsewhere ${ }^{6-12,35,42,47,48}$, an understanding of the direction of the shift seems to depend on knowledge of which sex gains relatively more by being large. Whether the problem is the sex ratio to be produced by a wasp constrained to rear a small offspring or the 'choice' to develop into a small adult male or female imposed on a nematode in a crowded host ${ }^{44.45}$, the principles underlying the force of natural selection remain the same.

Probably the largest gap in our knowledge is a clear demonstration of which sex gains relatively more by being large (except perhaps for some protogynous fish ${ }^{6,7,46}$, where it seems fairly clear that males gain more). Because of the difficulty in measuring fitness with sufficient precision to test whether females gain more by being large, we propose here an alternative experiment. The data suggest that males also gain by being larger. If a laboratory culture can arrange for females not to gain fitness by being larger, the selection pressures will be to reverse the sex ratio shift. The culture conditions will then favour males in larger hosts, females in smaller.

E.L.C. thanks J. Bull and J. Werren for many years of discussion on problems of sex allocation. Ken Sandlan, J. Bull, Richard Green, J. van Ierse, John Endler and D. Davidson read and improved the paper. E.L.C. and W.T.J. were supported by grants from the US NSF. J. v.d. A. and R.L.L-d.H. were supported by the Netherlands Organization for the Advancement of Pure Science (ZWO), grant 89-177.

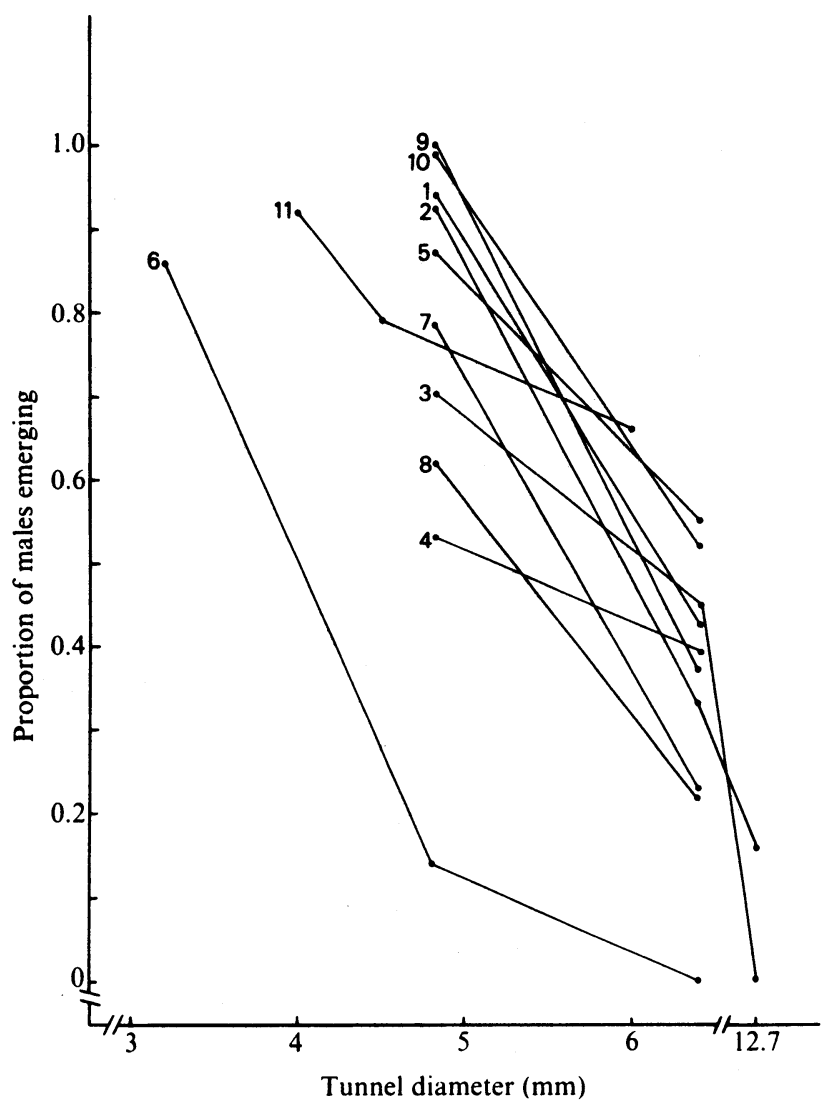

Fig. 6 Sex ratio at emergence related to tunnel diameter of nesting material for trap-nesting Hymenoptera. Data points refer to diameter of nesting material used in sampling. Lines connecting points are simply to keep species distinct. All sex ratio shifts are statistically significant at the 0.05 level (one-tailed, Fisher exact test) or better. Species 1-10 are from ref. 38, species 11 from ref. 39. Species as follows: 1, Euodynerus foraminatus; 2 , Euodynerus megaera; 3, Euodynerus schwarzi; 4, Pachodynerus erynnis; 5 , Ancistrocerus antilope ; 6, Ancistrocerus tigris ; 7, Symmorphus canadensis ; 8 , Tryarpilum tridentatum (North Carolina); 9, Tryarpilum johannis; 10 , Osmia lignaria ; 11, Megachile rotundata. Species $1-9$ are wasps, 10 and 11 are bees. 